\title{
Does a Mediterranean Model of Family Functioning in the Perception of Italian and Spanish Adolescents Exist? A Cross-National Study
}

\author{
Chiara Laudani ${ }^{1}$ \\ Giovanni Guzzo 1 \\ Valentina Lo Cascio² \\ Ugo Pace ${ }^{1}$ \\ Marco Cacioppo ${ }^{1}$ \\ ${ }^{1}$ Faculty of Human and Social Sciences, University Kore of Enna \\ Cittadella Universitaria, 94100, Enna, Italy \\ Email: ugopax@gmail.com,Tel. +39 935 536536; \\ 2Department of Economics, Business and Statistics, University of Palermo
}

Doi:10.5901/mjss.2014.v5n1p377

Abstract

The current study explored the influence of belonging to a different areas, South of Italy and Spain, on perception of parenting and family functioning adolescents in order to highlight the similarities and differences among two samples and verify if Mediterranean model of family exists". Empirical evidence of the existence of a Mediterranean family model has been conducted to verify which common and differentiating features exist in the perception of family ties among adolescents. Data highlighted that adolescents belonging to these areas report similar levels in different component of familial ties that can be defined as "Links and Roles": On the contrary, important differences were underlined on the perception of levels of rigidity and enmeshment within family: on the base of Marcia's model of identity status, we called this dimension "Foreclosure status".

Keywords: Family functioning, Adolescence, Mediterranean Model, Cultural Differences

\section{Introduction}

Traditional sociological and anthropological cross-cultural studies have underlined that the concept of family in Mediterranean societies that has been transmitted for a long time is based on two ideas: joint possession and dependence, such that the house is the center and meeting place for all members of the family who are linked together by a strong bond and mutual recognition of the value of family culture (Davies, 1977; Smith, 1981). In traditional Mediterranean society, the family is conceived as the cornerstone of life for individuals who perform the different roles of father, mother, child, and spouse. In this traditional society, the individual is inscribed within the family and the community. The idea of a trans-national model of family, such as the Mediterranean model, has been well illustrated by Rowland (1987) who suggested abandoning national borders as useful boundaries to delimit areas of research for the story of the family. In their place, he proposed the need for development of an analysis of the regional variables that are better able to show correlations, leading to the formulation of models. In this sense, some studies could be conducted in Italy, Spain, and Portugal which each have two clearly differentiated major regions: a northern region, where nuclear, conjugal, or restricted family structures are more frequent, and a southern region, where extended, parental, or enlarged families are predominant (Arriscado Nunes, 1987; Bevilacqua, 1993; Rowland, 1987). In this sense, as historiographical and anthropological research has advanced, Italy and Spain can be considered as part of an area-including Greece and Portugal, marked by a "different vein", which Micheli (2011) called strictly "southern European Mediterranean". According to Coleman (2002), changing the perspective from a country to a sub-country level of analysis is important. As a matter of fact, it is true that a country-level analysis is built on the coincidences of culture and language within national boundaries and with the common influences on the demographic behavior of uniform national systems. Nevertheless, between the north and south in both Spain and Italy, the organization of the family exhibits differences which clash with this presumed homogeneity. Even earlier, in the 1960s, Arensberg (1963) observed that rather than being Spanish, the 
Andalusians are better classified with Sicilians and others such as Mediterraneans, while the Gallegos and the Basques are better classified with others in the north, such as the people of the Atlantic fringe (p. 79). In this sense, the existence of more general psychosocial rules have taken shape and affected communities living within large areas of southern Europe (Reher, 1998). In these cross-national regions: "La famiglia" is at the center of life; it is not only a convenient way to provide for the proper raising of children but also the society's basic mode of organization. The individual without family is anomic; groups larger than "la famiglia" are secondary. (Bell, 1979, p. 211). Despite the large number of speculative studies conducted in the tradition of social sciences, there is little empirical evidence of the existence of Mediterranean family models. In this study, the most recent cross-cultural studies on family systems will be presented and a comparison between the perception of the characteristics of the family system of two Mediterranean countries, Italy and Spain, will be conducted to verify which common and differentiating features exist in the perception of family ties among adolescents.

\subsection{Cultural Diversity in Family Ties across Europe}

In Mediterranean countries, the term family indicates a diffuse network of relationships, obligations, and loyalties. From a historical perspective, one may distinguish three broad European culture areas (Jordan, 1988): (a) northwestern and central Europe, where, as a consequence of the specific characteristics of the rural economy, family members lived at growing distances; (b) eastern and southeastern Europe, where complex family structures, including three-generation families, were common; and (c) southern Europe, where family bonds were especially tight, although extended family patterns were not very common. Reher (1998) drew an even simpler dividing line, between central/northern Europe and the Mediterranean region, to distinguish "regions where traditionally the family group has had priority over the individual, and others where the individual and individual values have had priority over everything else" (p. 203).

More specifically, boundaries between households and kin networks are often thought of not only in emotional and affective terms, but also in legal and practical ones (Naldini, 2005). In a pioneering study on divergences and convergences in the European family, Roussel (1992) found that it was possible distinguish two cultural groups across Europe: Sweden, Norway, Finland and Denmark in the north; Portugal, Spain, Italy, and Greece in the south. In northern Europe, fertility was relatively high, divorce and cohabitation were high, and births out of marriage were strong or medium. Comparatively, in Spain, Italy, Portugal, and Greece, fertility, divortiality, cohabitation, and births out of marriage were all low. Moreover, in these countries, household size is much larger and a high percentage of the population lives in expanded households. In fact, in their late teens or early twenties, many people live with their parents, either full-time or part-time, and more and more people in their late twenties or even thirties are beginning to do so as well (Beyers \& Goossens 2008; Seiffge-Krenke, 2006). Not only do late adolescents in these countries often live with their parents, they also rely on their parents as important sources of support (Dotterer, Hoffman, Crouter, \& McHale, 2008; Furman \& Buhrmester, 1992). Due to the lack of support and care from the state, the individual is dependent on the support of his or her family, and within this economic dependency, a psychological dependency also develops. As such, economic dependency promotes the development of psychological dependency (Dwairy \& Achoui, 2010). Moreover, individuals in southern European countries are emotionally dependent on their families and adopt the values and attitudes of their families (Dwairy, 1998). The definitive departure of young people tends to coincide more or less with their marriages and finding stable jobs. The years between adolescent maturity and marriage are spent largely within the parental household. Generally, marriage does not even enter the picture unless it is accompanied by the corresponding emancipation from the parental home and the formation of a new household. The same can be said about older people, who often move in with their children, particularly when their pensions are low. All these characteristics represent what has been defined in human sciences as "The Mediterranean model of family" (Reher, 1998).

\subsection{Culture, Family and Adolescence}

Within the psychological literature, several researchers have underlined how different cultures are characterized by relatively "strong" or relatively "weak" family ties, especially in regards to the perception of adolescents (Sven Reher, 1998). Authors have examined the family's role and functions in adolescence in various countries (Schwarz, 2012), enabling them to identify the intercultural frameworks that explain both continuities and dissimilarities in parenting across different social contexts (Claes et al., 2011). Understanding the whole family as a unit is important to an adolescent's development, in that it is the medium through which a variety of developmental functions unfold (Beyers \& Gossens, 2008; Stuart \& Jose, 2012). During this phase of the life cycle, family relationships undergo important changes that can be considered to be a result of young people's beginning to seek their autonomy (Phinney et al., 2005). This evolutional task is characterized by a process of co-evolution (Onnis, 2010): parents and adolescents are engaged together in a 
developmental challenge (Minuchin, 1974) that will lead to a transformation of intergenerational ties (Scabini \& Cigoli, 2006). According to family system model, the adequate family functioning in adolescence will occur when the hierarchical structure within families contains subsystems organized by communicative rules that enhance harmonious decisionmaking and the formation of firm, yet flexible boundaries (Lindahl et al., 2004; Minuchin, 1974). Several researchers have found that those familial features play a fundamental role in adolescents' developmental trajectories (Pace, \& Zappulla, 2010; Guzzo, Lo Cascio, \& Pace, 2013).

A key theoretical and methodological issue in current cross-cultural psychology is the necessity for determining context variables and cultural variables, which are related to psychological variables, and which can explain universals and differences in psychological variables related to cultural dimensions (Georges, 2001). Therefore, it may be valid to consider a cultural dimension when studying individual and family functioning issues.

\subsection{Aims of the present study}

Starting from previous findings, the current study explored various family variables, such as parental bonds, or general functioning, comparing and contrasting data from samples belonging to two different southern regions of Italy and Spain, Sicilia and Andalusia. In a nutshell, this study specifically explored the influence of culture on perception of parenting and family functioning adolescents in order to highlight the similarities and differences among two samples and verify if Mediterranean model of family exist.

\section{Research Method}

\subsection{Participants}

The study examined 171 Italian students (75 boys and 96 girls) who attended two universities in the south of Italy and 169 Spanish students (70 boys and 99 girls) who attended two universities in the south of Spain. We selected the schools as being representative of the socioeconomic structure and cultural differences of both country contexts, based on local census data. Italian and Spanish undergraduate students (340) ranging in age from 18 to 23 were recruited. In particular, the average age of the Italian students was 20.25 (SD = 1.80 years), whereas among Spanish students, the average age was $19.66(S D=1.67$ years). Both the Italian and Spanish samples consisted only of Caucasian students. Moreover, the samples were comparable in terms of years and types of schooling, sex, and age group composition. Approximately $92 \%$ of the Italian and Spanish participants lived in two-parent households, whereas the remainder of participants lived with only one parent (usually the mother).

\subsection{Procedure}

Participants completed consecutively self-report measures on family functioning and parental psychological control. They also provided information on their age, gender, and ethnicity prior to completing questionnaires. Researchers collected data during school visits conducted between 2011 and 2012. In particular, data were collected in two separate waves (data from Italy were collected first, then Spanish data). Research team travelled to each site to collect data. Respondents completed the questionnaires independently during regular school time. Anonymity was assured as there were no names or other identification used. Respondents sealed the questionnaires and returned them to the researchers at the time of data collection. Letters describing the purpose and content of the study were distributed among the adolescents' who were invited to participate in the study. A standard informed assent was obtained before answering the questionnaire, and students were informed that they could stop participation at any time. Research procedures described in this article were performed in compliance with the American Psychological Association, the Italian Psychological Association ethical guidelines for research and the ethical guidelines of the Spanish Psychological Society.

\subsection{Measure}

Dependency-oriented and Achievement oriented Psychological Control Scale (DAPCS; Soenens et al. 2010; Italian version Guzzo et al., 2013). It was administered to assess adolescents' perceived parental psychological control. The scale consists of 16 items concerning parental dependency-oriented psychological control (DPC; 8 items; e.g., "My 
parents are only friendly with me if I rely on them instead of on my friends") and achievement-oriented psychological control (APC; 8 items; e.g., "My parents make me feel guilty if my performance is inferior"), which participants were asked to answer on a 5-point Likert scale ranging from 1 (strongly disagree) to 5 (strongly agree). The original English version of the questionnaire was translated into Spain and then back-translated by a native speaker to ensure its comparability with the English version.

Family Assessment Device (FAD; Epstein et al., 1983; Italian version, Roncone et al., 1998; Spanish version, Barroilhet et al., 2009). It was administered to assess the adolescents' perception of family functioning. It consists of 60 items concerning seven dimensions of family functioning: Problem-solving (e.g., "Resolution of most everyday problems around the house"), Communication (e.g., "Saying things instead of hinting at them"), Roles (e.g., "Making sure members meet family responsibilities"), Affective Responsiveness (e.g., "Ability to cry openly"), Affective Involvement (e.g., "Paying interest to others when something is important to them"), Behavior Control (e.g., "Knowledge of what to do in an emergency"), and General Functioning (e.g., "Reciprocal support in times of crisis"). Participants were asked to answer on a 4-point Likert scale, ranging from 1 (strongly disagree) to 4 (strongly agree). The internal consistency reliability is ranging from .72 to .92 (Epstein et al., 1983).

Parental Bonding Instrument (PBI; Parker, Tupling, \& Brown, 1979; Italian version, Scinto et al., 1999; Spanish version, Gomez-Beneyto et al., 1993). It is a 25-item self-report measure designed to assess parent-child relationships using a Likert scale from 0 (very unlike) to 3 (very unlike). Twelve of the items are Care items (e.g., "Appeared to understand my problems and worries"), which allows for a maximum score of 36 for the Care dimension, and thirteen of the items are the Overprotection items (e.g. "Tried to make me feel dependent on him"), which allows for a maximum score of 39 for the Overprotection dimension. Care involves a dimension from parental affection, warmth and empathy (high scores) to parental coldness, indifference and rejection (low scores). Overprotection or control ranges from intrusiveness to infantilization (high scores) through to the detached promotion of independence (low scores). The PBI has good psychometric properties and is insensitive to the effects of the respondent's mood (Parker, 1989).

Family Adaptability and Cohesion Evaluation Scales (FACES-IV; Olson, 2011; Italian version, Baiocco et al., 2012; Spanish version, Rivero et al., 2010). It measures the family functioning using six scales: two balanced scales, Cohesion (sample item: "Family members are supportive of each other during difficult times") and Flexibility (sample item: 'My family is able to adjust to change when necessary'), assessing central-moderate areas and four unbalanced scales, Enmeshed (sample item: "Family members feel guilty if they want to spend time away from the family"), Disengaged (sample item: "Family members seem to avoid contact with each other when at home"), Chaotic (sample item: "Our family feels hectic and disorganized"), and Rigid (sample item: "There are clear consequences when a family member does something wrong"), assessing the upper and the lower extremes of Cohesion and Flexibility respectively (Olson \& Gorall 2006). It is hypothesized that the central or balanced levels make for optimal family functioning, while the extremes or unbalanced levels are generally seen as problematic for relationship over the longer term. There are seven items in each scale, making a total of 42 items. The participants are asked to rate how much they agree with the statement on a 5-point Likert scale ranging from 1 (strongly disagree) to 5 (strongly agree). The six scales in FACES IV were found to be reliable and valid: the Cronbach's alpha coefficients ranged from .63 to .73 (Baiocco et al., 2012). There are seven items in each scale, making a total of 42 items. The participants are asked to rate how much they agree with the statement on a 5-point Likert scale ranging from 1 (strongly disagree) to 5 (strongly agree). The six scales in FACES IV were found to be reliable and valid: the Cronbach's alpha coefficients ranged from .63 to .73 (Baiocco et al., 2012).

\section{Analysis Result}

We conducted preliminary analyses, including descriptive statistics and gender differences in the key study variables (ANOVAs). Moreover, a Multiple Correspondence Analysis (MCA) was performed in order to examine the perception adolescents have of family functioning and their mothers' and fathers' parenting. Family functioning and mothers' and fathers' parenting were entered as active variables, while country was introduced as supplementary variables in order to not affect the MCA solution, but still be able to know how the categories of this variable are positioned on the correspondence map. MCA is a special technique of exploratory factor analysis that graphically displays multivariate categorical data (Benzécri, 1992). Seen as a generalization of principal component analysis for categorical variables, it allows researchers to analyze the pattern of relationships in a complex data matrix by replacing raw data with a simpler data matrix (Abdi \& Valentin, 2007). 
Associations between variables are examined by calculating the chi-square distance between variable modalities and between individuals. These associations can then be represented graphically by plotting the projection of the rows and the columns onto the dimensions extracted by the factor analysis. If two categories have similar count patterns, their profiles will be closer together in the correspondence map and they will have closer co-ordinates on dimensions that account for most of the variance (Benzécri, 1973). In summary, we selected MCA because it stems from a statistical strategy that makes it possible to simultaneously manage multiple variables within a given system. It has the advantage of plotting the similarities and differences across the countries on a comprehensive graphic.

To conduct the MCA, continuous data for each of the variables of the study were reorganized into three categories: these categories represent values within 1 (low) and 3 (high). For instance, "Problem solving 3" includes ratings above the third quartile on the problem solving scale of FAD, whereas "Problem solving 1" includes ratings in the first quartile. Results will be limited to the interpretation of the first two factors extracted by the MCA (Ben Ammou \& Saporta, 2003). Moreover, additional analyses were done to control the potential effect of disparities between the subsamples. We computed the MCA on SPSS software. Descriptive analyses for all independent and dependent variables are presented in Table 1. In order to analyze possible gender differences in the key study variables, we performed oneway analyses of variance (ANOVAs). They revealed no statistically significant gender effects in the scores of all variables in the study.

Fig. 1 illustrates the MCA two-dimensional space that represents family functioning and mothers' and fathers' parenting as active variables, while country as supplementary variables. MCA results are presented as follows: (1) interpretation of axes using contributions of the active variables to each factor; (2) interpretation of the position of the supplementary variable with respect to each factor; and (3) interpretation based on interpoint proximities in the twodimensional map.

The first axis $\left(\lambda_{1}\right)$ extracted by MCA, with an eigenvalue of $\lambda_{1}=6.38$ and $30.38 \%$ of inertia explained, bipolarly encompasses the following measures of family functioning at the negative pole: high levels of disengaged and cahotic, low levels of cohesion, flexibility, problem solving, communication, roles, affective responsiveness, affective involvement, behavior control, and general family functioning.

Moreover, it encompasses the following measures of parenting: high levels of paternal and maternal dependencyoriented psychological control, paternal and maternal achievement-oriented psychological control, paternal and maternal overprotection, and low levels of paternal and maternal care.

At the opposite positive pole, the first axis encompasses the following measures of family functioning: low levels of disengaged and cahotic, high levels of cohesion, flexibility, problem solving, communication, roles, affective responsiveness, affective involvement, behavior control, and general family functioning.

Moreover, it encompasses the following measures of parenting: low levels of paternal and maternal dependencyoriented psychological control, paternal and maternal achievement-oriented psychological control, paternal and maternal overprotection, and high levels of paternal and maternal care. Thus, the first axis includes parenting and family functioning dimensions ranging from bad family functioning and dysfunctional parenting to good family functioning and functional parenting. Hence, this axis represents weak links and undefined roles of family, as opposed to strong links and defined roles of family.

The second axis $\left(\lambda_{2}\right)$, with an eigenvalue of $\lambda_{2}=2.87$ and $13.68 \%$ of inertia explained, bipolarly groups low levels of rigid and enmeshed at the positive pole and high levels of rigid and enmeshed at the negative pole. This second axis includes dimensions ranging from low amount of emotional closeness and high level of decisiveness to extreme amount of emotional closeness and low level of decisiveness.

Hence, this axis represents achievement of separateness of family, as opposed to foreclosure status of family. As for differences between countries, the two country-related profiles of categories are not significantly different from the mean profile of the first axis [t(171) $=-0.96 ; p>.05$ for Italy and $\mathrm{t}(169)=0.98 ; p>.05$ for Spain], but they are significantly different from the mean profile for the second axis [t(171) $=2.99 ; p<.05$ for Italy and $t(169)=2.95 ; p<.05$ for Spain]. Compared to Spain adolescents' perception, Italian adolescents perceive higher levels of rigidity and enmeshment in their family functioning. Italian and Spain adolescents report similar levels in all scales including in the first axis.

Fig. 1. Two-dimensional space by MCA for family functioning and maternal and paternal practices. 


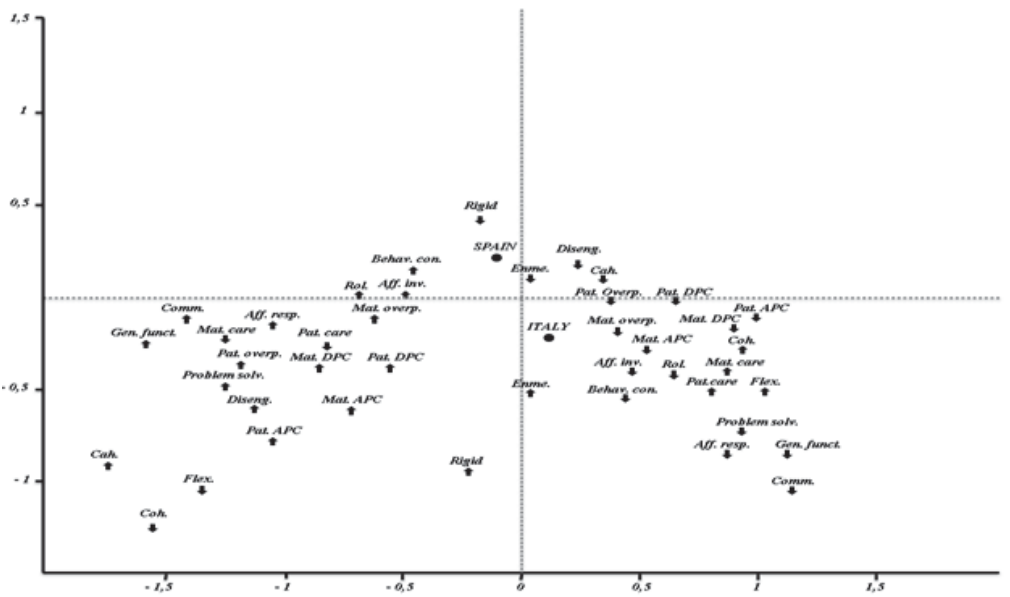

Notes: $\downarrow$ represents value 1 (low) and $\uparrow$ represents level 3 (high) for each of the variables of the study; Diseng. = Disengaged; Cah. = Cahotic; Coh. = Cohesion; Flex. = Flexibility; Problem solv. = problem solving; Comm. = Communication; Rol. = Roles; Aff. Resp. = affective responsiveness; Aff. Inv. = affective involvement; Behav. Con. = behavior control; Gen. funct. = general family functioning; Rigid = rigid; Enme. = Enmeshed; Pat. DPC = paternal dependency-oriented psychological control; Mat. DPC = maternal dependency-oriented psychological control; Pat. APC = paternal achievement-oriented psychological control; Mat. APC = maternal achievement-oriented psychological control; Pat. overp. $=$ paternal overprotection; Mat. overp $=$ maternal overprotection; Pat. care $=$ paternal care; Mat. care $=$ maternal care .

\section{Discussion and Conclusions}

The purpose of this study was to explore the influence of culture on adolescents' perceptions of parenting and family functioning in order to highlight the similarities and differences among two different southern regions in Italy and Spain. In particular, the fundamental question motivating the present research was: Does a Mediterranean model of family exist? In fact, during these years, testing the so-called Mediterranean model has become one of the prime goals of research for family researchers working on southern Europe (Viazzo, 2003). Nevertheless, the results are controversial.

Regarding the primary analyses, even though we did not predict gender differences with explicit hypotheses, the present study allowed us to make several interesting observations. In fact, our data revealed no statistically significant gender effects in the scores of all variables in the study. A possible explanation is that during late adolescence, both boys and girls have achieved similar independence and balance from their family, and as a consequence, they are paired to emphasize the aspects of the family functioning. However, data concerning gender differences in adolescents' perceptions of family functioning and parenting are not univocal. Several studies have highlighted that adolescent girls demonstrate heightened emotional awareness in family relationships (Laghi, Baiocco, Lonigro, Capacchione, \& Baumgartner, 2012; Pace \& Zappulla, 2013), while another study revealed no significant gender differences in different dimensions of family functioning, except for a higher level of self-disclosure with parents as reported by the girls (Bandura et al., 2011). Interesting findings emerged from the Multiple Correspondence Analyses (MCA) that detects two axes structuring the parental practices and family functioning perceived by all adolescents in the two countries. Particularly, present data highlighted that adolescents belonging to Italy and Spain report similar levels in all scales included in the first axis, which are disengaged and chaotic, cohesion, flexibility, problem-solving, communication, roles, affective responsiveness, affective involvement, behavior control, and general family functioning. Moreover, the axis encompasses the following measures of parenting: paternal and maternal dependency-oriented psychological control, paternal and maternal achievement-oriented psychological control, paternal and maternal overprotection, and paternal and maternal care. and Roles."

Due to the number, the importance and pervasiveness of the shared characteristics, this axis was called "Links

This result is in accord with sociological and anthropological cross-cultural studies that support the issue of the local specificity of family in the Mediterranean regions (Reher, 1998) and provides one of the still few pieces of empirical evidence regarding the existence of Mediterranean family model. On the contrary, regarding what the second axis is concerned with, important differences were underlined: inspired by Marcia's model of identity status; we called it 
"Foreclosure status." In fact, compared to their Spanish peers, Italian adolescents perceive higher levels of rigidity and enmeshment in their family functioning. The data therefore highlight how, despite a general agreement about the perception of family functioning in the two compared regions, the important difference, with respect to any characteristic deemed crucial to the adaptation of the system and for individuals who are part of it, cannot be disregarded (Cacioppo, Pace, \& Zappulla, 2013; Minuchin, Rosman, \& Baker, 1978). Accordingly, within a general perceptual uniformity of family functioning across the two regions, it seems that there is a specificity of the Italian culture in structuring mutual relations in which emotional closeness and loyalty are demanded, and consequently, the development of the personal individuation may be hampered by a sort of foreclosing family system. These results are in line with other studies (Manzi et al., 2006) that underscored the notion that the Italian family is characterized by a high degree of emotional bonding and support from both parents, yet also by restrictions and by relations of enmeshment-this also lends support to other observations regarding the presence and maintenance of adolescents' requirements and restrictions within the Italian family (Lanz, 2000).

Based on the idea of a trans-national model of family, such as the Mediterranean model, on the basis of which to delimit areas of research for the story of the family, abandoning national borders as useful boundaries, the present findings underline the need for developing the analysis of regional variables that are better able to show correlations, leading to the formulation of models. In fact these results show that the set of real or perceived "Links and Roles" that determine family functioning provides a comparative basis from which we can better understand the confluence of determinants that constitute the basis of cultural differences in which family ties assume a similar value, as in the case in Sicily and Andalusia, or a different value, as is the case, presumably, in other parts of Europe. Nonetheless, the data highlight that there is a certain degree of difference affecting families and family life within single societies. We may suppose that the perception of southern Italian adolescents of a "Foreclosure family" can hit heavy walls of conflict when the natural healthy questioning of adolescents breaks with the idea of family in which components are very dependent on each other (enmeshed) or committed to maintaining the status quo and denying the need for change (rigid). These results are in line with the framework in mainstream psychology that considers developmental processes and structures to be fundamentally similar, although it recognizes that culture can influence human development to accomplish objectives that foster context-specific adaptation. Thus, though parenting differences in neighboring cultures can be subtle, they nonetheless represent instances of specific adaptation.

Bukowski and Sippola (1998) refer to this as "local knowledge modeling": this notion ascribes the power to create different developmental goals to culture, though it considers the fundamental developmental processes and structures to be universal. This multiplicity of forms and behavior, however, does not negate the existence of more general regularities affecting large areas of Europe.

\subsection{Strengths and Limitations}

The main strength of this study is to support the low empirical evidence regarding the existence of a Mediterranean family model, comparing and contrasting data from samples belonging to two different southern regions in Italy and Spain. However, the mentioning of the limitations of our study should not be omitted. All the obtained information was collected by self-report measures; as such, the accuracy of individual reporters cannot be assured. Social desirability can in fact also affect the results. The measurements used may also introduce some measurement biases across cultures and across age groups. Moreover, self-assessment is indispensable in order to be able to measure perceptions of parenting and family functioning statuses. Future research should strive to conduct longitudinal studies examining the developmental trajectories of adolescents' perceptions of parenting and family functioning across diverse cultures. Finally, research should tap into a more diverse population, not belonging to Mediterranean regions, in order to compare cultural patterns of different families, which has been present for years in the theoretical models of the social sciences, but whose empirical evidence is still extremely scarce.

\section{References}

Abdi, H., \& Valentin, D. (2007). Multiple correspondence analysis. In N. J. Salkind (Ed.), Encyclopedia of measurement and statistics (pp. 651-657). Thousand Oaks (CA): Sage.

Arensberg, C.M. (1963). The old world peoples: The place of European cultures in world ethnography. Anthropological Quarterly, 36, 7599.

Arriscado Nunes, J. (1987). Nupcialidade e familia em Portugal (seculos XVI-XX). Balanço critico e perspectivas, Paper presented at $10^{\circ}$ Congrès Hispano-Luso-Italià de demografia histórica, Barcelona 
Baiocco, R., Cacioppo, M., Laghi, F. \& Tafà, M. (2012). Factorial and Construct Validity of FACES IV Among Italian Adolescents. Journal of Child and Family Studies, 21, 325-338.

Bandura, A., Caprara, G. V., Barbarelli, C., Regalia, C., \& Scabini, E. (2011). Impact of family efficacy beliefs on quality of family functioning and satisfaction with family life. Applied Psychology: An International Review, 60, 421-448.

Barroilhet, S., Cano-Prous, A., Cervera-Enguix, S., Forjaz, M. J., \& illén-Grima, F. (2009). A spanish version of the Family Assessment Device. Social Psychiatry \& Psychiatric Epidemiology, 44, 1051-1065.

Bell, R. M., (1979). Fate and Honor, Family and Village. Demographic and Cultural Change in Rural Italy since 1800. The University of Chicago Press, Chicago

Ben Ammou, S., \& Saporta, G. (2003). On the connection between the distribution of eigenvalues in multiple correspondence analysis and log-linear models. Revstat Statistical Journal, 1, 42-79.

Benzécri, J.P. (1973). L'analyse des données. II. L'analyse des correspondances (Data analyses II. Correspondence analysis). Paris: Dunod.

Benzécri, J.P. (1992). Correspondence analysis handbook. New York: Marcel Dekker.

Bevilacqua, P. (1993). Breve storia dell'Italia Meridionale dall'Ottocento ad oggi. Donzelli, Rome

Beyers,W., \& Goossens, L. (2008), Dynamics of perceived parenting and identity formation in late adolescence, Journal of Adolescence , 31, 165-184.

Bukowski, W.M., \& Sippola, L.K. (1998). Diversity and social mind: goals, construct, culture and development. Developmental Psychology, 34, 742-746.

Cacioppo, M., Pace, U., \& Zappulla, C. (2013). Parental psychological control, quality of family context and life satisfaction among Italian adolescents. Child Indicators Research, 6, 179-191.

Claes, M., Perchec, C., Miranda, D., Benoit, M., Bariaud, F., Lanz, M., Marta, E., \& Lacourse, E. (2011). Adolescents' perceptions of parental practices: A cross-national comparison of Canada, France, and Italy. Journal of Adolescence, 34, 225-238.

Coleman, D. (2002). Populations of the industrial world: A convergent demographic community? International Journal of Population Geography, 8, 319-344.

Dotterer, A.M., Hoffman, L., Crouter, A.C., \& McHale, S.M. (2008). A Longitudinal Examination of the Bidirectional Links Between Academic Achievement and Parent -Adolescent Conflict, Journal of Family Issues, 29, 762-779.

Dwairy, M. (1998). Cross-cultural counseling: The Arab-Palestinian case. New York: Haworth Press.

Dwairy, M., \& Achoui, M. (2010). Adolescents-Family Connectedness: A First Cross-Cultural Research on Parenting and Psychological Adjustment of Children. Journal Children Family Study 9, 8-15.

Epstein, N. B., Baldwin, L., \& Bishop, D. S. (1983). The McMaster family assessment device. Journal of Marital and Family Therapy, 9 , 171-180.

Furman, W., \& Buhrmester, D. (1992). Age and sex-differences in perceptions of networks of personal relationships. Child Development, $63,103-115$.

Georgas, J., Mylonas, K., Bafiti, T., Poortinga, Y. H., Christakopoulou, S., Kagitcibasi, C., et al. (2001). Functional relationships in the nuclear and extended family: A 16 culture study. International Journal of Psychology, 36, 289-300.

Gomez-Beneyto, M., Tomas, A.P., Aguilar, K., \& Leal, C. (1993). Psychometric properties of the parental boding instrument in a Spanish sample. Social Psychiatry and Psychiatric Epidemiology, 28, 252-5.

Guzzo, G., Lo Cascio, V., \& Pace, U. (2013). The Role of Individual and Relational Characteristics on Alcohol Consumption among Italian Adolescents: A Discriminant Function Analysis, Child Indicators Research, 6, 605-18.

Guzzo, G., Lo Cascio, V., Pace, U., \& Zappulla, C. (2013). Psychometric Properties and Convergent Validity of the DependencyOriented and Achievement-Oriented Psychological Control Scale (DAPCS) with Italian Adolescents. Journal of Child and Family Studies. 10.1007/s10826-013-9786-2

Jordan, T. G. (1988). The European culture area. New York: Harper \& Row.

Laghi, F., Baiocco, R., Lonigro, A., Capacchione, G., Baumgartner E. (2012). Family functioning and binge drinking among Italian adolescents. Journal of Health Psychology, 17, 1132-1141.

Lanz M. (2000). La ricerca empirica. L'accordo tra genitori e figli: un processo bidimensionale, in M. Lanz, E. Marta (a cura di), Cognizioni sociali e relazioni familiari (pp.17-53). Milano: Franco Angeli.

Lindahl, K. M., Malik, N.M., Kaczynski, K., \& Simons, S.J. (2004). Couple power dynamics, systemic family functioning, and child adjustment: A test of a mediational model in a multiethnic sample. Development and Psychopathology, 16, 609-630.

Manzi, C., Vignoles, V. L., Regalia, C., \& Scabini, E. (2006). Cohesion and enmeshment revisited: differentiation, identity, and well-being in two European cultures. Journal of Marriage and Family, 68, 673-689.

Micheli, G.A. (2012).Two Strong Families in Southern Europe? Re-Examining the Geography of Kinship Regimes Stemming from the Reciprocity Mechanisms Between Generations. Europe Journal Population, 28,17-38.

Minuchin, S. (1974). Families and family therapy. Cambridge, MA: Harvard University Press.

Minuchin, S., Rosman, B. L., \& Baker, L. (1980). Famiglie psicosomatiche: L'anoressia mentale nel contesto familiare. Roma: Astrolabio. Naldini, M., (2005). Le politiche sociali in Europa, Roma, Carocci.

Olson, D. (2011). Faces IV and The Circumplex Model: Validation Study. Journal of Marital and Family Therapy, 37, 1: 64-80.

Onnis, L. (Ed.). (2010). Creating bondings, healing bondings. Turin: Bollati Boringheri. Parker, G. (1989). The parental bonding instrument: Psychometric properties reviewed. Psychiatric Developments, 4, 317-335

Pace, U., \& Zappulla, C. (2010). Relations between suicidal ideation, depression, and emotional autonomy from parents in adolescence. 
Journal of Child and Family Studies, 19, 747-756.

Pace, U., \& Zappulla, C. (2013). Detachment from parents, problem behaviors, and the moderating role of parental support among Italian adolescents. Journal of Family Issues, 34, 768-783

Parker, G., Tupling, H., \& Brown, L. B. (1979). A parental bonding instrument. British Journal of Medical Psychology, 52, 1-10.

Phinney, J., Kim-Jo, T., Osorio, S., \& Vilhjalmsdottir, P. (2005). Autonomy and relatedness in adolescent-parent disagreements: Ethnic and developmental factors. Journal of Adolescent Research, 20, 8-39.

Reher, D. S. (1998). Family ties in western Europe: Persistent contrasts. Population and Development Review, 24, $203-234$.

Roncone, R., Rossi, L., Muiere, E., Matteucci, M., Giacomelli, R., Tonietti, G., et al. (1998). The Italian version of the Family Assessment Device. Social Psychiatry and Psychiatric Epidemiology, 33, 451-461.

Roussel, L. (1992). La famille en Europe Occidental; divergences and convergences, Population, 1, 133-152.

Rowland, R. (1987). Nupeialidade, familia, mediterraneo, Bollettino di Demografia Storica, 5.

Scabini, E., \& Cigoli, V. (2006). Family identity: ties, symbols, and transitions. Mahwah, NJ: Erlbaum.

Schimmenti, A. (2012). Unveiling the hidden self: Developmental trauma and pathological shame.

Psychodynamic Practice, 18, 195-211.

Schwarz, B., Mayer, B., Trommsdorff, G., Ben-Arieh, A., Friedlmeier, M., Lubiewska, K., Mishra, R., \& Peltzer, K. (2012). Does the Importance of Parent and Peer Relationships for Adolescents' Life Satisfaction Vary Across Cultures? Journal of Early Adolescence 32, 55-80.

Scinto, A, Marinangeli, M.G., Kalyvoka, A., Daneluzzo, E., Rossi, A. (1999). Utilizzazione della versione italiana del Parental Bonding Instrument (PBI) in un campione clinico ed in un campione di studenti: uno studio di analisi fattoriale esplorativa e confermatoria. Epidemiologia e Psichiatria Sociale, 8, 276-283.

Smith, R. M. (1981). The people of Tuscany and their families in the fifteenth century: Medieval or Mediterranean? Journal of Family History, 6, 107-128.

Seiffge-Krenke, I. (2006). Leaving home or still in the nest? Parent-child relationships and psychological health as predictors of different leaving home patterns. Developmental Psychology, 42, 864-876.

Soenens, B., Vansteenkiste, M., \& Luyten, P. (2010). Toward a domain-specific approach to the study of parental psychological control: distinguishing between dependency-oriented and achievement-oriented psychological control, Journal of Personality, 78, 217256.

Stuart, J., \& Jose, P. E. (2012). The Influence of Discrepancies Between Adolescent and Parent Ratings of Family Dynamics on the Well-Being of Adolescents. Journal of Family Psychology, 1-12.

Viazzo, P. P. (2003). What's so special about the Mediterranean? Thirty years of research on household and family in Italy. Continuity and Change, 18, 111-137. 
